\title{
A THEOREM ON AMENABLE SEMIGROUPS
}

\author{
BY \\ EDMOND GRANIRER
}

Introduction. A semigroup $G$ is said to have a left [right] invariant mean, in other words is left [right] amenable, if there exists a linear functional $\phi$ on the space of all the bounded real functions on $G$ (with the sup. norm), $m(G)$, which satisfies for each $f \in m(G)$ and $a \in G$

$$
\begin{aligned}
\inf _{g \in G} f(g) & \leqq \phi(f) \leqq \sup _{g \in G} f(g), \\
\phi(f) & =\phi\left(l_{a} f\right), \quad\left[\phi(f)=\phi\left(r_{a} f\right)\right],
\end{aligned}
$$

where $\left(l_{a} f\right)(g)=f(a g)$ and $\left(r_{a} f\right)(g)=f(g a)$.

An invariant mean is a right and left invariant mean. $M l(G),[M r(G)] \subset m(G)^{*}$ will denote the set of left [right] invariant means and $\operatorname{dim} M l G=n$ will mean that the linear manifold spanned by $M l(G) \subset m(G)^{*}$ is $n$-dimensional (see [5, $\S 2]) . Q: l_{1}(G) \rightarrow m(G)^{*}$ will denote the natural mapping of the semigroup algebra $l_{1}(G)$ into $m(G)^{*}$.

The following is a result of I. S. Luthar (see [9]):

A commutative semigroup $G$ has a unique invariant mean (i.e., $\operatorname{dim} M l(G)=1$ ) if and only if $G$ contains a finite ideal. Luthar's proof also yields that the unique invariant mean has to belong to $Q l_{1}(G)$.

The main result of this paper is the following:

THEOREM 1. If $G$ is a semigroup with $\operatorname{dim} M l(G)=n$ and $\operatorname{dim} \operatorname{Mr}(G)=m$ where $0<m, n<\infty$ then:

$$
m=n=1, \quad M l(G)=M r(G) \subset Q l_{1}(G)
$$

and $G$ contains a finite group which is a two-sided ideal (and therefore a minimal left and minimal right ideal).

If $G$ contains a finite group which is a two-sided ideal then it is also easily shown that $M l(G)=M r(G) \subset Q l_{1}(G)$ and $\operatorname{dim} M l(G)=\operatorname{dim} M r(G)=1$.

Theorem 1 implies that for commutative semigroups $G$ only one of the two cases can occur: either $\operatorname{dim} M l(G)=1$ or $\operatorname{dim} M l(G)=\infty$. It also implies Luthar's result.

We have the following remarks to Theorem 1 :

Presented to the Society, January 26, 1963; received by the editors December 3, 1962 and, in revised form, March 1, 1963. 
For countable semigroups much more than Theorem 1 is proved by this author in [5] (see [5, Corollary (5.5) and Remark (3.1)]), i.e.:

If $G$ is a semigroup which satisfies that $\operatorname{dim} \operatorname{Ml}(G)=n$ and $\operatorname{Mr}(G) \neq \varnothing$ then $n=1, M l(G)=\operatorname{Mr}(G) \subset Q l_{1}(G)$ and $G$ contains a finite group which is a twosided ideal.

In particular if $G$ is countable and has a unique left invariant mean $\mu$, then as easily seen $\mu$ is also a right invariant mean, but moreover, by the above theorem, $\mu$ is the unique right invariant mean and $G$ contains a finite group which is a twosided ideal.

Thus for countable semigroups $\operatorname{dim} \operatorname{Mr}(G)<\infty$ is not imposed on $G$ while we get the same result as in Theorem 1 of this paper. Therefore $\operatorname{dim} \operatorname{Mr}(G)<\infty$ is seemingly a superfluous condition and this author was not yet able to drop it. A proof of Theorem 1 of this paper without imposing on $G$ the condition $\operatorname{dim} \operatorname{Mr}(G)<\infty$ but only $\operatorname{Mr}(G) \neq \varnothing$ would lead to a proof of Theorem A in [5] without imposing on $G$ to be countable (see $[5, \S 1]$ ), i.e., to a complete characterization of left amenable semigroups with a finite dimensional set of left invariant means.

There are in this paper several other results which may have some interest for their own sake. For instance, it is shown as an immediate consequence of Theorem 1 that for commutative semigroups $G$, the radical of the second conjugate algebra $m(G)^{*}$, is infinite dimensional, if $G$ does not contain finite ideals. If we take $G$ to be an infinite commutative group then, as known, the group algebra $l_{1}(G)$ is semisimple (i.e., has zero radical) and $Q l_{1}(G)$ is $w^{*}$ dense in the second conjugate algebra $m(G)^{*}$ (as defined in [3, p. 526]). Nevertheless one gets that the radical of $m(G)^{*}$ is infinite dimensional (which is a conjecture made by P. Civin and B. Yood; see [1, p. 853] and also [5, last page "added in print"']).

Some definitions and notations. Let $G_{0} \subset G$ be a semigroup and subsemigroup and let $\pi: m(G) \rightarrow m\left(G_{0}\right)$ such that $(\pi x)(g)=x(g)$ for $g \in G_{0}, l_{a}: m(G) \rightarrow m(G)$ such that $\left(l_{a} x\right)(g)=x(a g)$ for $a, g \in G, l_{a}^{0}: m\left(G_{0}\right) \rightarrow m\left(G_{0}\right)$ such that $\left(l_{a}^{0} x\right)(g)=x(a g)$ for $a, g \in G_{0}$.

If $\phi, \psi \in m(G)^{*}$ then $\phi \odot \psi \in m(G)^{*}$ will be defined for $x \in m(G)$ by $(\phi \odot \psi)(x)=\phi(y)$ where $y(b)=\psi\left(l_{b} x\right)$. (See Day [3, p. 527].)

If $A \subset G$ and if $1_{A}$ is the characteristic function of $A$ and if $\phi \in m(G)^{*}$ then we will write sometimes $\phi(A)$ instead of $\phi\left(1_{A}\right)$. We will denote by $J l(G)=\left\{\phi \in m(G)^{*}\right.$; $L_{g} \phi=\phi$ for $\left.g \in G\right\}$ (which is the set of left invariant elements of $G$ ).

For other notations we refer to $[5, \S 2]$. We will prove now

LEMMA 1. Let $G$ be a left amenable semigroup and $\mu \in M l(G)$. If $G_{0} \subset G$ is a subsemigroup such that $\mu\left(G_{0}\right)>0$ then the linear operator $T: m\left(G_{0}\right)^{*} \rightarrow m(G)^{*}$ defined by $T v=\mu \odot\left(\pi^{*} v\right)($ see Day $[3, p .533])$ satisfies $T\left[M l\left(G_{0}\right)\right] \subset M l(G)$ and

$$
\left\|T v_{0}\right\| \geqq \mu\left(G_{0}\right)\left\|v_{0}\right\| \text { for any } v_{0} \in J l(G) \text {. }
$$


Proof. If $v \in m(G)^{*}$ then $T v=\mu \odot\left(\pi^{*} v\right) \in J l(G)$ (see proof of Corollary 2 in Day [3, p. 529]). If $v \in m\left(G_{0}\right)^{*}$ is a mean then so is $\pi^{*} v$ (see [6, Remark (2.2)]) and therefore so is $\mu \odot \pi^{*} v$ (if $\phi, \psi$ are means then so is $\phi \odot \psi$ as is easily seen). Therefore $T\left[M l\left(G_{0}\right)\right] \subset M l(G)$. Since $\odot$ is distributive (see [3, p. 527]) and $\pi^{*}$ linear, $T$ is linear.

We will prove now that for any $\varepsilon>0$

$$
\left\|T v_{0}\right\| \geqq \mu\left(G_{0}\right)\left[\left\|v_{0}\right\|-\varepsilon\right] .
$$

Let $x_{0} \in m\left(G_{0}\right)$ be such that

$$
\left\|x_{0}\right\|=1 \text { and } v_{0}\left(x_{0}\right) \geqq\left\|v_{0}\right\|-\varepsilon>0 .
$$

We define now $x \in m(G)$ by:

$$
x(g)=x_{0}(g) \text { for } g \in G_{0} \text { and } x(g)=0 \text { for } g \notin G_{0} .
$$

Obviously $\|x\|=1$ and:

$$
\left[T v_{0}\right](x)=\left[\mu \odot\left(\pi^{*} v_{0}\right)\right](x)=\mu(y)
$$

where $y$ is defined by:

$$
y(h)=\left[\pi^{*} v_{0}\right]\left(l_{h} x\right)=v_{0}\left(\pi l_{h} x\right) .
$$

By Remark (2.3) in [6] $y(h)$ is constant on left cosets of $G$ with respect to $G_{0}$. Since $G_{0}$ is left amenable we have for any $a, b \in G_{0}, a G_{0} \cap b G_{0} \neq \varnothing$, as easily seen [5, end of proof of Corollary (5.5)]. This yields the existence of $g_{1}, g_{2}$ in $G_{0}$ satisfying $a g_{1}=b g_{2}$. Thus $a \approx b$ (see for instance definition after Lemma (5.1) in [5]), which shows that $G_{0}$ is included in exactly one coset of $G$ with respect to $G_{0}$ (let it be $H_{0}$ ).

If $a \notin H_{0}$ then $a G_{0} \cap G_{0}=\varnothing$ (otherwise $a b=c$ for $b, c$ in $G_{0}$ and thus $a \approx c$, i.e., $a \in H_{0}$ ).

We claim now that $y(g)=v_{0}\left(x_{0}\right) \cdot 1_{\mathbf{H}_{0}}(g)$.

By the above remark $y(g)$ is constant on $H_{0}$ and if $a \in G_{0} \subset H_{0}$ then:

$$
y(a)=v_{0}\left(\pi l_{a} x\right)=v_{0}\left(l_{a}^{0} \pi x\right)=v_{0}(\pi x)=v_{0}\left(x_{0}\right) .
$$

(For the second equality see Remark (2.1) in [6]. The third holds since $v_{0} \in J l\left(G_{0}\right)$.) Thus $y(g)=v_{0}\left(x_{0}\right)$ for any $g \in H_{0}$. If now $a \notin H_{0}$ then we have for $g$ in $G_{0}$

$$
\left[\pi\left(l_{a} x\right)\right](g)=\left(l_{a} x\right)(g)=x(a g)=0
$$

since $a G_{0} \cap G_{0}=\varnothing$ (see the definition of $x$ ). Therefore

$$
y(a)=v_{0}\left(\pi l_{a} x\right)=v_{0}(0)=0
$$

which proves that

However

$$
y(g)=v_{0}\left(x_{0}\right) 1_{H_{0}}(g) .
$$




$$
\begin{aligned}
{\left[T v_{0}\right](x) } & =\mu(y)=\mu\left[v_{0}\left(x_{0}\right) 1_{H_{0}}\right]=v_{0}\left(x_{0}\right) \mu\left(H_{0}\right) \geqq v_{0}\left(x_{0}\right) \mu\left(G_{0}\right) \\
& \geqq \mu\left(G_{0}\right)\left[\left\|v_{0}\right\|-\varepsilon\right]
\end{aligned}
$$

which shows that

$$
\left\|T v_{0}\right\| \geqq \mu\left(G_{0}\right)\left\|v_{0}\right\|,
$$

thus finishing the proof of our lemma.

Remark 1. This lemma proves in particular that the above constructed $T$ when restricted to $\operatorname{Jl}\left(G_{0}\right)$ is a linear, one to one operator which maps $M l\left(G_{0}\right)$ into $M l(G)$. We get thus one part of the

COROLlaRY 1. If $G_{0}$ is a subsemigroup of the left amenable semigroup $G$ with $\operatorname{dim} M l(G)<\infty$ and if for some $\mu \in M l(G), \mu\left(G_{0}\right)>0$ then:

$$
0<\operatorname{dim} M l\left(G_{0}\right) \leqq \operatorname{dim} M l(G) .
$$

Proof. In order to get the whole corollary we have only to remark that by Day $\left[3\right.$, p. 518], $M l\left(G_{0}\right) \neq \varnothing$ which shows that $\operatorname{dim} M l\left(G_{0}\right)>0$. (Notice that $0 \notin M l\left(G_{0}\right)$ since the zero function of $m(G)^{*}$ is not a mean.)

Remark 2. Corollary 1 is also true when "left amenable" is replaced by "right amenable" and $\mu \in M l(G)$ is replaced by $\mu \in \operatorname{Mr}(G)$.

To see this, and in fact this way one could prove that all the theorems of $[5 ; 6]$ remain true when "left" is replaced by "right," we define in $G$ a new multiplication, i.e., $a \triangle b=b a$. This multiplication renders $G$ a semigroup, let us call it $G^{\prime}$, which is left amenable if $G$ was right amenable and moreover $\operatorname{Mr}(G)=\operatorname{Ml}\left(G^{\prime}\right)$. Thus if $G_{0} \subset G$ and $\mu \in M r(G)$ are such that $\mu\left(G_{0}\right)>0$ then $\mu \in M l\left(G^{\prime}\right)$ will satisfy that $\mu\left(G_{0}^{\prime}\right)>0$ where $G_{0}^{\prime}$ is $G_{0}$ with the new multiplication $\Delta$. Thus by Corollary 1

$$
0<\operatorname{dim} M r\left(G_{0}\right)=\operatorname{dim} M l\left(G_{0}^{\prime}\right) \leqq \operatorname{dim} M l\left(G^{\prime}\right)=\operatorname{dim} M r(G),
$$

which shows that this remark holds true.

Remark 2 and Corollary 1 prove:

COROLlARY 2. If $G$ is an amenable semigroup such that

$$
\operatorname{dim} M l(G)<\infty \text { and } \operatorname{dim} M r(G)<\infty
$$

and if $G_{0} \subset G$ is a subsemigroup such that $\mu\left(G_{0}\right)>0$ for some

$$
\mu \in \operatorname{Ml}(G) \cap \operatorname{Mr}(G)
$$

hen

$$
0<\operatorname{dim} M l\left(G_{0}\right) \leqq \operatorname{dim} M l(G)
$$

and

$$
0<\operatorname{dim} \operatorname{Mr}\left(G_{0}\right) \leqq \operatorname{dim} M r(G) .
$$


REMARK. If $G_{0} \subset G$ are as in Lemma 1 then, $T\left[\left(M l\left(G_{0}\right)\right]=M l(G)\right.$ is not necessarily true. In fact if $G=\left\{\alpha_{0}, \alpha_{1}, \cdots, \alpha_{n}\right\}$ with $\alpha_{i} \alpha_{j}=\alpha_{j}$ for $0 \leqq i, j \leqq n$ and if $G_{0}=\left\{\alpha_{0}\right\}$ then $\operatorname{dim} \operatorname{Ml}\left(G_{0}\right)=1$ while $\operatorname{dim} M l(G)=n+1$ (see examples on the last page of [6]).

DEFINITION 1. (a) If $G$ is a semigroup then we define the following relation between elements of $G: a(r) b$ if and only if there exists $h$ in $G$ such that $a h=b h$.

(b) A relation $\sim$ between elements of a semigroup will be called a two-sided stable equivalence if it is an equivalence and if $a \sim b$ implies $c a \sim c b$ and $a c \sim b c$ for any $c \in G$ (see $[10$, p. 60]).

LemMa 2. If $G$ is a left amenable semigroup then the relation $a(r) b$ of Definition 1 (a) is a two-sided stable equivalence.

Proof. $G$ is left amenable and therefore the intersection of any two right ideals is nonvoid [5, end of proof of Corollary (5.5)]. If $a \in G, a(r) a$ holds and if $a, b \in G$ satisfy $a(r) b$ then $b(r) a$ holds as is easily seen. Let now $a, b, c \in G$ satisfy $a(r) b$ and $b(r) c$; then by definition there are $h_{1}, h_{2}$ in $G$ such that $a h_{1}=b h_{1}$ and $b h_{2}=c h_{2}$. But $h_{1} G \cap h_{2} G \neq \varnothing$; therefore there are $h^{\prime}, h^{\prime \prime}$ in $G$ such that $h_{1} h^{\prime}=h_{2} h^{\prime \prime}$. Thus:

$$
\left(a h_{1}\right) h^{\prime}=\left(b h_{1}\right) h^{\prime}=b\left(h_{1} h^{\prime}\right)=b\left(h_{2} h^{\prime \prime}\right)=\left(b h_{2}\right) h^{\prime \prime}=\left(c h_{2}\right) h^{\prime \prime}=c h_{1} h^{\prime}
$$

which shows that $a(r) c$. We proved thus that the above relation is an equivalence.

If now $a(r) b$ then $a h_{0}=b h_{0}$ for some $h_{0} \in G$ which implies that $(c a) h_{0}=(c b) h_{0}$, in other words that $c a(r) c b$ for any $c \in G$. It remains to show that $a c(r) b c$ holds also for any $c \in G$. By the above $c G \cap h_{0} G \neq \varnothing$; therefore there are $g^{\prime}, g^{\prime \prime}$ such that $c g^{\prime}=h_{0} g^{\prime \prime}$. Thus $a c g^{\prime}=a h_{0} g^{\prime \prime}=b h_{0} g^{\prime \prime}=b c g^{\prime}$ which shows that $a c(r) b c$. This finishes the proof of Lemma 2.

REMARK 3. If $(r)$ is a two-sided stable relation in a semigrcup $G$, then let $\bar{G}=G /(r)$ stand for the set whose elements are all the equivalence classes of elements of $G$ with respect to the relation $(r)$. Let us define the mapping $\phi: G \rightarrow G$ by $\phi(a)=\bar{a}$ where $\bar{a}$ is the equivalence class which contains the element $a \in G$ (in other notation $\bar{a}=\{g \in G ; g(r) a\})$. A multiplication between the elements of $\bar{G}$ can be defined: $\bar{a} \circ \bar{b}=\bar{a} \bar{b}$, i.e., $\bar{a} \circ \bar{b}$ is the (r) equivalence class of elements of $G$ which contains the element $a b \in G$.

It is well known (see [10, pp. 361-362]) that the above defined multiplication o is well defined and associative rendering thus $\bar{G}$ a semigroup, provided that $(r)$ is a two-sided stable equivalence relation. The above defined $\phi: G \rightarrow \bar{G}$ such that $\phi(a)=\bar{a}$ is, by its definition, a homomorphism of $G$ onto $\bar{G}$. Please remember that the elements of $\bar{G}$ are subsets of $G$ and we will write sometimes for some $\bar{a} \in \bar{G}$ that $\bar{a} \subset G$. The function $1_{\bar{a}}$ will then be defined as

$$
1_{\bar{a}}(g)=\left\{\begin{array}{l}
1 \text { for } g \in \bar{a}, \\
0 \text { for } g \notin \bar{a} .
\end{array}\right.
$$


$1_{\bar{a}}$ will then denote a function from $G$ to the reals and not from $\bar{G}$ to the reals. This way, if $\psi \in m(G)^{*}$, then $\psi\left(1_{\bar{a}}\right)=\psi(\bar{a})$ where $\bar{a} \subset G$. This is used in places where no ambiguity can arise.

Lemma 3. Let $G$ be an amenable semigroup such that $\operatorname{dim} \operatorname{Mr}(G)<\infty$. Then $\bar{G}=G /(r)$ (where $(r)$ is the relation of Definition $1(\mathrm{a}))$ is a finite group.

Proof. $G$ is amenable and a fortiori left amenable therefore by Lemma 2 the relation $a(r) b$, which holds if and only if there exists $h \in G$ such that $a h=b h$, is a two-sided stable equivalence. By Remark $3 G=G /(r)$ is a semigroup which is a homomorphic image of $G$. By Luthar's theorem (for proof see [3, pp. 531-532]) there is a linear operator $F^{*}: m(G)^{*} \rightarrow m(\bar{G})^{*}$ such that $F^{*}[\operatorname{Mr}(G)]=\operatorname{Mr}(\bar{G})$, therefore, $\operatorname{dim} \operatorname{Mr}(\bar{G}) \leqq \operatorname{dim} \operatorname{Mr}(G)<\infty$. But $\bar{G}$ is a semigroup with right cancellation since $\bar{a} \circ \bar{c}=\bar{b} \circ \bar{c}$ implies $\overline{a c}=\overline{b c}$ which implies $a(c h)=b(c h)$ for some $h \in G$, which by definition means that $a(r) b$, in other words that $\bar{a}=\bar{b}$. But $\bar{G}$ as a homomorphic image of $G$ is right amenable and left amenable (see Day [3, p. $515(C)]$ ). Thus $G$ is a right amenable semigroup with right cancellation and with $\operatorname{dim} \operatorname{Mr}(\bar{G})=n<\infty$. We can apply Theorem $\mathrm{E}$ of [6] (which is true when "left" is replaced by "right") and get that $\bar{G}$ is finite and is the union of $n$ finite disjoint groups each of which is a right ideal. Since $\bar{G}$ is also left amenable the intersection of any two right ideals is nonvoid and therefore $n=1$ and $\bar{G}$ is a finite group, which finishes the proof of this lemma.

In what follows a slight generalization of a theorem of I. S. Luthar (see [9, p. 41, Lemma 1]) will be needed. The proof follows closely Luthar's proof:

THEOREM OF I. S. LUTHAR. If $E$ is a directed set such that for any $e \in E$ there exists $e^{\prime} \in E$ with $e^{\prime}>e$ and $e^{\prime} \neq e$ and if $E$ does not contain a finite cofinal set then $E$ contains $n$ cofinal disjoint sets for any $0<n<\infty$.

REMARK. It is important to notice that $E$ is a directed set as defined in Kelley [7, p. 65], i.e., $e_{2} \geqq e_{1}$ and $e_{1} \geqq e_{2}$ do not necessarily imply $e_{1}=e_{2}$. The definition of a directed set in Köthe [8,p. 9], requires that $e_{1} \geqq e_{2}$ and $e_{2} \geqq e_{1}$ implies $e_{1}=e_{2}$ and the set to which Luthar's theorem will be applied, in what follows, will not have in general this property.

Proof. If $A \subset E$ is a finite subset then there is an element $e \in E, e \notin A$ and $e \geqq a$ for each $a \in A$. Otherwise each $e \in E$ which satisfies $e \geqq a$ for each $a \in A$ would belong to $A$. If $e \in E$ is arbitrary, then let $f \in E$ be such that $f \geqq e$ and $f \geqq a$ for each $a \in A$. This implies that $f \in A$ in other words, that $A$ is a cofinal set which contradicts our assumptions since $A$ is finite.

Let now $e_{1} \in E$. There is then some $e_{2} \in E$ such that $e_{2} \geqq e_{1}$ and $e_{2} \neq e_{1}$. If $\left\{e_{1}, \cdots, e_{k}\right\}$ were chosen as distinct elements which satisfy $e_{i} \geqq e_{j}$ for $1 \leqq j \leqq i$ and $i=1,2, \cdots, k$ then by the above we can choose $e_{k+1} \notin\left\{e_{1}, \cdots, e_{k}\right\}$ such that $e_{k+1} \geqq e_{i}$ for $1 \leqq i \leqq k$. We choose this way a sequence of distinct elements $\left\{e_{i}\right\}$ which satisfies $e_{1}<e_{2}<e_{3}<\cdots$. 
Let now $n>1$ be a fixed integer and $\mathscr{U}$ be the set of all the $n+1$ tuples $\left(A_{1}, A_{2}, \cdots, A_{n}, F\right)$ where $F$ is a subset of $E$ and $A_{i}, 1 \leqq i \leqq n$, are maps from $F$ to $E$ which satisfy the following:

(L1) $A_{i}(F) \cap A_{j}(F)=\varnothing$ for $i \neq j, 1 \leqq i, j \leqq n$.

(L2) $A_{i}(F)$ and $A_{j}(F)$ are cofinal in each other for $1 \leqq i, j \leqq n$.

(L3) $A_{i}(F)$ is cofinal in $F$ for each $1 \leqq i \leqq n$.

We prove now that $\mathscr{U}$ is not empty. Let us take $F$ as the sequence $\left\{e_{i}\right\}$ we chose above and let

$$
A_{i}^{0}\left(e_{j}\right)=e_{(j-1) n+i}
$$

Thus:

$$
\begin{gathered}
A_{1}^{0}(F)=\left\{e_{k n+1} ; k=0,1,2, \cdots\right\}, A_{2}^{0}(F)=\left\{e_{k n+2} ; k=0,1,2, \cdots\right\}, \\
\cdots, A_{n}^{0}(F)=\left\{e_{k n+n} ; k=0,1,2, \cdots\right\} .
\end{gathered}
$$

It is easy to check that the here chosen $n+1$ tuple $\left(A_{1}^{0}, \cdots, A_{n}^{0}, F\right)$ satisfies conditions (L1), (L2), (L3), which shows that $\mathscr{U} \neq \varnothing$. Let us partial order $\mathscr{U}$ by $\left(A_{1}, A_{2}, \cdots, A_{n}, F\right) \leqq\left(A_{1}^{\prime}, A_{2}^{\prime}, \cdots, A_{n}^{\prime}, F^{\prime}\right)$ which holds if and only if $F \subset F^{\prime}$ and for each $1 \leqq i \leqq n, A_{i}$ is the restriction of $A_{i}^{\prime}$ to $F$.

If now $\mathscr{A}=\left\{\left(A_{1}^{\alpha}, \cdots, A_{n}^{\alpha}, F^{\alpha}\right) ; \alpha \in I\right\}$ is a linearly ordered subset of $\mathscr{U}$ then we define $\left(A_{1}, \cdots, A_{n}, F\right)$ to be: $F=\bigcup_{\alpha \in I} F^{\alpha}$ and if $e \in F$ then $e \in F^{\alpha}$ for some $\alpha$ and then let $A_{i}(e)=A_{i}^{\alpha}(e)$ for $1 \leqq i \leqq n$. If $e \in F^{\beta}$ for some $\beta \neq \alpha$ then since $\left(A_{1}^{\alpha}, \cdots, A_{n}^{\alpha}, F^{\alpha}\right)$ and $\left(A_{1}^{\beta}, \cdots, A_{i}^{\beta}, F^{\beta}\right)$ are comparable we get that $A_{i}^{\alpha}(e)=A_{i}^{\beta}(e)$, which shows that $\left(A_{1}, \cdots, A_{n}, F\right)$ is well defined.

Now $A_{i}(F)=\bigcup_{\alpha \in I} A_{i}^{\alpha}\left(F^{\alpha}\right)$, thus $A_{i}(F) \cap A_{j}(F) \neq \varnothing$ would imply $A_{i}^{\alpha}\left(F^{\alpha}\right) \cap A_{j}^{\beta}\left(F^{\beta}\right) \neq \varnothing$ for some $\alpha, \beta \in I$. But either $\left(A_{1}^{\alpha}, \cdots, A_{n}^{\alpha}, F^{\alpha}\right) \geqq\left(A_{1}^{\beta}, \cdots, A_{n}^{\beta}, F^{\beta}\right)$ or $\left(A_{1}^{\beta}, \cdots, A_{n}^{\beta}, F^{\beta}\right) \geqq\left(A_{1}^{\alpha}, \cdots, A_{n}^{\alpha}, F^{\alpha}\right)$ and thus either $A_{j}^{\beta}\left(F^{\beta}\right) \subset A_{j}^{\alpha}\left(F^{\alpha}\right)$ and then $A_{i}^{\alpha}\left(F^{\alpha}\right) \cap A_{j}^{\alpha}\left(F^{\alpha}\right) \neq \varnothing$ or $A_{i}^{\alpha}\left(F^{\alpha}\right) \subset A_{i}^{\beta}\left(F^{\beta}\right)$ and then $A_{i}^{\beta}\left(F^{\beta}\right) \cap A_{j}^{\beta}\left(F^{\beta}\right) \neq \varnothing$, both of which cannot be. This proves that $\left(A_{1}, \cdots, A_{n}, F\right)$ satisfies condition (L1). Moreover $A_{i}(F), A_{j}(F)$ are cofinal in each other, since if $e \in A_{j}(F)$ then $e \in A_{j}^{\alpha}\left(F^{\alpha}\right)$ but, then there is an $e^{\prime} \in A_{i}^{\alpha}\left(F^{\alpha}\right) \subset A_{i}(F)$ which satisfies $e^{\prime} \geqq e$. If now $e \in F$ then $e \in F^{\alpha}$ for some $\alpha$ and there is an $e^{\prime} \in A_{i}^{\alpha}\left(F^{\alpha}\right) \subset A_{i}(F)$ such that $e^{\prime} \geqq e$, which proves that the above chosen $\left(A_{1}, \cdots, A_{n}, F\right)$ is an element of $\mathscr{U}$. But obviously $\left(A_{1}, \cdots, A_{n}, F\right)$ is an upper bound for the linearly ordered set $\mathscr{A}$. Zorn's lemma yields the existence of a maximal element in $\mathscr{U}$.

If $\left(A_{1}, \cdots, A_{i}, F\right)$ is such a maximal element then we shall prove that $A_{i}(F)$ is a cofinal set in $E$ for each $1 \leqq i \leqq n$ and thus finish the proof of this theorem.

Let us first notice that either $A_{i}(F)$ is cofinal in $E$ for each $1 \leqq i \leqq n$ or $A_{i}(F)$ is not cofinal in $E$ for any $1 \leqq i \leqq n$. This follows from the fact that $A_{j}(F), A_{i}(F)$ are cofinal in each other.

Suppose now that $A_{i}(F)$ are not cofinal in $E$ for any $1 \leqq i \leqq n$. Then there exist $a_{1}, \cdots, a_{n}$ in $E$ such that $e \geqq a_{i}$ for $1 \leqq i \leqq n$ implies $e \notin \bigcup_{i=1}^{n} A_{i}(F)$. 
If $e_{1} \geqq a_{i}$ for $1 \leqq i \leqq n$ then we can construct as above a sequence of distinct elements $e_{1}<e_{2}<\cdots<e_{n-1}<e_{n} \cdots$ and since $A_{j}(F)$ are cofinal in $F$ it follows that $e_{i} \notin F$ (otherwise let $e_{k} \in F$; then there is an $e \in A_{i}(F)$ such that $e \geqq e_{k}$ which cannot be).

We define now $F^{*}=F \cup\left\{e_{i} ; i \geqq 1\right\}$ and

$$
\begin{aligned}
& A_{i}^{*}(f)=A_{i}(f) \text { for } f \text { in } F, \\
& A_{i}^{*}\left(e_{j}\right)=A_{i}^{0}\left(e_{j}\right)=e_{(j-1) n+i} ;
\end{aligned}
$$

see (4). $\left(A_{1}^{*}, \cdots, A_{n}^{*}, F^{*}\right)$ is an element of $\mathscr{U}$ and obviously

$$
\left(A_{1}^{*}, \cdots, A_{n}^{*}, F^{*}\right)>\left(A_{1}, \cdots, A_{n}, F\right)
$$

(and the sides do not equal), which cannot be since $\left(A_{1}, \cdots, A_{n}, F\right)$ was maximal. Thus $A_{1}(F), \cdots, A_{n}(F)$ are all cofinal in $E$ and disjoint.

We will prove now the main theorem of this paper:

THEOREM 1. Let $G$ be a semigroup such that $\operatorname{dim} M l(G)=n$ and $\operatorname{dim} \operatorname{Mr}(G)$ $=m$ where $0<m, n<\infty$. Then $m=n=1, \operatorname{Ml}(G)=M r(G) \subset Q l_{1}(G)$ and $G$ contains a finite group which is a two-sided ideal (and therefore a minimal right ideal and a minimal left ideal).

Proof. If ( $r$ ) is the relation of Definition 1(a) then by Lemma 3, $\bar{G}=G /(r)$ is a finite group whose elements will be $\bar{e}, \bar{a}_{1}, \cdots, \bar{a}_{k-1}$, where $\bar{e}$ is the identity element and $\left\{e, a_{1}, \cdots, a_{k-1}\right\} \subset G$ is a set of fixed representatives all over this proof.

We have to remember that the elements of $\bar{G}$ are also subsets of $G$. If $\bar{a}_{i} \in \bar{G}$ then $\bar{a}_{i}^{-1}$ will be inverse of $\bar{a}_{i}$ in the finite group $\bar{G}$. Fix now $i$ and let $a$ be some element of $\bar{a}_{i}^{-1} \subset G$.

Then we can write when looking upon $\bar{e}$ as a subset of $G$ and $1_{\bar{e}}$ as belonging to $m(G)$ that

$$
l_{a} 1_{\bar{e}}(g)=1_{\bar{e}}(a g) \geqq 1_{\bar{a}_{i}}(g)
$$

since if $g \in \bar{a}_{i}$ then $\overline{a g}=\bar{a} \circ \bar{g}=\bar{a}_{i}^{-1} \circ \bar{a}_{i}=\bar{e}$ and thus agree (see R. Thibault's lecture in [11]) which implies equality of both sides. While if $g \notin \tilde{a}_{i}$ then the right side is zero while the left one non-negative. Thus if $\phi \in M l(G) \cap \operatorname{Mr}(G)$ then:

$$
\phi\left(1_{\bar{e}}\right)=\phi\left(l_{a} 1_{\bar{e}}\right) \geqq \phi\left(1_{\bar{a}_{i}}\right) .
$$

If now $b \in \bar{a}_{i}$ then

$$
l_{b} 1_{\bar{a}_{i}}(g)=1_{\bar{a}_{i}}(b g) \geqq 1_{\bar{e}}(g)
$$

since for $g \in \bar{e} \subset G$ we have $\overline{b g}=\bar{b} \circ \bar{g}=\bar{a}_{i} \circ \bar{e}=\bar{a}_{i}$ and thus $b g \in \bar{a}_{i}$. Therefore and thus

$$
\phi\left(1_{\bar{a}_{i}}\right)=\phi\left(l_{b} 1_{\bar{a}_{i}}\right) \geqq \phi\left(1_{\bar{e}}\right)
$$




$$
\phi(\bar{e})=\phi\left(1_{\bar{e}}\right)=\phi\left(1_{\bar{a}_{i}}\right)=\phi\left(\bar{a}_{i}\right) .
$$

But $\bar{e}, \bar{a}_{1}, \cdots, \bar{a}_{k-1}$ are $n$ disjoint subsets of $G$ whose union is $G$ (any $g \in G$ is $(r$ ) equivalent to some $a_{i}$ or $e$ ). Therefore

$$
1=\phi(G)=\phi(\bar{e})+\phi\left(\bar{a}_{1}\right)+\cdots+\phi\left(\bar{a}_{k-1}\right)=k \phi(\bar{e})
$$

which shows that $\phi(\bar{e})=1 / \mathrm{l} k>0$.

But $\bar{e} \subset G$ is a subsemigroup since if $a, b \in \bar{e}$ then $\overline{a b}=\bar{a} \circ \bar{b}=\bar{e} \circ \bar{e}=\bar{e}$, i.e., $a b \in \bar{e}$.

By applying Corollary 2 we get that $0<\operatorname{dim} M l(\bar{e}) \leqq n$ and $0<\operatorname{dim} M r(\bar{e}) \leqq m$.

Let now $a, b \in \bar{e}$, then $a(r) b$ and thus there is a $c \in G$ such that $a c=b c$. But if $c \in \bar{a}_{i}$ for some $i$ and then if $d \in \bar{a}_{i}^{-1}$ we have $a c d=b c d$ and $\overline{c d}=\bar{c} \circ \bar{d}=\bar{a}_{i} \circ \bar{a}_{i}^{-1}=\bar{e}$ which shows that $c d \in \bar{e}$. Thus $\bar{e}$ is a subsemigroup of $G$ which has the property that for any $a, b \in \bar{e}$ there is $f \in \bar{e}$ such that $a f=b f$.

We claim now that it is sufficient, in order to finish the proof of our theorem, to show that $\bar{e}$ contains an element $e_{0}$ with the property: for any $d \in \bar{e}$ with $d \neq e_{0}$ one has also $d e_{0}=e_{0}$. Let us assume that we have proved already its existence. Then if $a \in G$ then $a \in \bar{e}$ or $a \in \bar{a}_{i}$ for some $1 \leqq i \leqq k-1$. If $a \in \bar{e}$ and $a \neq e_{0}$ then $a e_{0}=e_{0}$ which implies $a e_{0}^{2}=e_{0} e_{0}$. If $\left[e_{0}\right]$ will denote the subsemigroup generated by $e_{0} \in G$ then this equality means that $a$ is in the same left coset of $G$ with respect to $\left[e_{0}\right]$ as $e_{0}$. If now $a \in \bar{a}_{i}$ then there is some $c \in G$ such that $a c=a_{i} c$. If $c \in \bar{e}$ then either $c=e_{0}$ and then $a e_{0}=a_{i} e_{0}$, or $c \neq e_{0}$ and then $a e_{0}=a c e_{0}=a_{i} c e_{0}=a_{i} e_{0}$. And if $c \in \bar{a}_{j}$ then $c d \in \bar{e}$ for any $d \in \bar{a}_{j}^{-1}$ and thus if $c d=e_{0}$ then $a e_{0}=a c d$ $=a_{i} c d=a_{i} e_{0}$ and if $c d \neq e_{0}$ then $a e_{0}=a\left[(c d) e_{0}\right]=(a c)\left(d e_{0}\right)=a_{i}\left[(c d) e_{0}\right]$ $=a_{i} e_{0}$. Thus in any case $a e_{0}=a_{i} e_{0}$ which shows that $a$ is in the same left coset of $G$ with respect to $\left[e_{0}\right]$ as $a_{i}$. In other words $G$ contains a countable subsemigroup which is $\left[e_{0}\right]$, such that the set of left cosets of $G$ with respect to $\left[e_{0}\right]$, is a finite set ( $\left[e_{0}\right]$ is even a finite subsemigroup). We can apply now Corollary (5.5) and remark (5.3), both of [5], to get that $m=n=1, M l(G)=M r(G) \subset Q l_{1}(G)$ and that $G$ contains exactly one finite group which is also a left ideal (and therefore a minimal left ideal) and exactly one finite group and right ideal (and therefore a right minimal ideal), which coincide.

Thus in order to finish the proof of our theorem we will prove following theorem which in fact is the more difficult part of this paper:

THEOREM 2. Let $G$ be a semigroup which satisfies

(a) for each $a, b \in G$ there is a $c \in G$ such that $a c=b c$;

(b) $\operatorname{dim} M l(G)=n, 0<n<\infty$.

Then $G$ has an element $e_{0}$ with the property that for any $g \in G$ such that $g \neq e_{0}, g e_{0}=e_{0}$.

Proof. Let $\left\{g_{1}, \cdots, g_{k}\right\}$ be a finite subset of $G$. Then there are $h_{1}, \cdots, h_{k-1}$ in $G$ such that $g_{i} h_{i}=g_{i+1} h_{i}$ for $1 \leqq i \leqq k-1$. But any element $h$ of the right ideal $h_{i} G$ 
satisfies also $g_{i} h=g_{i+1} h$. Since $G$ is left amenable $(0<n)$ the intersection of any two, and by an easy induction, the intersection of any finite number of right ideals is nonvoid. Therefore, if $h_{0} \in \bigcap_{i=1}^{k-1} h_{i} G$ then $g_{1} h_{0}=g_{2} h_{0}=g_{3} h_{0}=\cdots$ $=g_{k} h_{0}$.

Let now $\left\{\phi_{\alpha}\right\}$ be a net of finite means in $m(G)^{*}$ which converges strongly to left invariance (see Day [3, p. 524]). If $\left\{g_{\alpha}\right\}$ is any net of elements in $G$ defined on the same directed set as $\left\{\phi_{\alpha}\right\}$ then $\left\{R_{g_{a}} \phi_{\alpha}\right\}$ is also a net of finite means which converges strongly to left invariance (this is a result of Day $[3$, p. 526]) since

$$
\begin{aligned}
\left\|L_{g} R_{g_{-}} \phi_{\alpha}-R_{g_{\nu}} \phi_{\alpha}\right\| & =\left\|R_{g_{-}} L_{g} \phi_{\alpha}-R_{g_{\alpha}} \phi_{\alpha}\right\| \leqq\left\|R_{g_{\alpha}}\right\|\left\|L_{g} \phi_{\alpha}-\phi_{\alpha}\right\| \\
& \leqq\left\|L_{g} \phi_{\alpha}-\phi_{\alpha}\right\| \rightarrow 0 .
\end{aligned}
$$

Let us chose the $g_{\alpha}$ 's as follows: if $\phi_{\alpha}=\sum_{i=1}^{k} \beta_{i} Q 1_{a_{l}}\left(k, \beta_{1}, \cdots, \beta_{k}\right.$ and $a_{1}, \cdots, a_{k}$ depend on $\alpha$ ) then there is a $g_{\alpha} \in G$ such that

$$
a_{1} g_{\alpha}=a_{2} g_{\alpha}=\cdots=a_{k} g_{\alpha}=b_{\alpha} .
$$

For this $g_{\alpha}$ we will have

$$
R_{g_{.}} \phi_{\alpha}=\sum_{1}^{k} \beta_{i} R_{g_{\nu}} Q 1_{a_{i}}=\sum_{1}^{k} \beta_{i} Q 1_{a_{i} g_{\nu}}=\sum_{1}^{k} \beta_{i} Q 1_{b_{\alpha}}=Q 1_{b_{\alpha}} .
$$

(Since $R_{b}\left(Q 1_{a}\right) f=\left(Q 1_{a}\right)\left(r_{b} f\right)=\left(r_{b} f\right)(a)=f(a b)=\left(Q 1_{a b}\right) f$ for $f \in m(G)$ and $\sum_{1}^{k} \beta_{i}=1$.)

Thus the net of finite means $\left\{Q 1_{b_{x}}\right\}$ is converging strongly to left invariance, i.e., for any $g \in G$

$$
\lim _{\alpha}\left\|L_{g} Q 1_{b_{.}}-Q 1_{b_{\alpha}}\right\|=\lim _{\alpha}\left\|Q 1_{g b_{\alpha}}-Q 1_{b_{\alpha}}\right\|=\lim _{\alpha}\left\|1_{g b_{\alpha}}-1_{b_{\alpha}}\right\|=0
$$

and the last norm is in $l_{1}(G)$. But $\left\|1_{a}-1_{b}\right\|=2$ if and only if $a \neq b$ and $\left\|1_{a}-1_{b}\right\|=0$ if and only if $a=b$ where these norms are in $l_{1}(G)$. Therefore for any $g_{0} \in G$ there is an $\alpha_{0}$ (which depends on $g_{0}$ ) such that: $\alpha \geqq \alpha_{0}$ implies $\left\|1_{g_{0} b_{.}}-1_{b_{\text {.. }}}\right\|<2$, i.e., $\left\|1_{g_{0} b_{\sim}}-1_{b_{\alpha}}\right\|=0$. In other words for any $g_{0} \in G$ there is an $\alpha_{0}$ such that

$$
\alpha \geqq \alpha_{0} \text { implies } g_{0} b_{\alpha}=b_{\alpha} .
$$

We introduce now a partial order relation in $G$ which will render $G$ a directed set (according to the definition in Kelley [7, p. 65] (not as in [8, p. 9])). If $a, b \in G$ then $b \geqq a$ if either $a=b$ or $a b=b$.

Let $b \geqq a$ and $c \geqq b$. If either $a=b$ or $b=c$ then obviously $c \geqq a$. If neither is the case then $b c=c$ and $a b=b$ which implies that $a c=(a b) c=b c=c$ and thus $c \geqq a$ which shows that $c \geqq b$ and $b \geqq a$ imply $c \geqq a$.

If now $a, b \in G$ then there are by (5) $\alpha_{1}, \alpha_{2}$ such that $\alpha \geqq \alpha_{1}$ implies $a b_{\alpha}=b_{\alpha}$ and $\alpha \geqq \alpha_{2}$ implies $b b_{\alpha}=b_{\alpha}$. If $\alpha_{0} \geqq \alpha_{1}$ and $\alpha_{0} \geqq \alpha_{2}$ then $a b_{\alpha_{0}}=b_{\alpha_{0}}$ and $b b_{\alpha_{0}}=b_{\alpha_{0}}$, 
i.e., $b_{\alpha_{0}} \geqq a$ and $b_{\alpha_{0}} \geqq b$. Thus $G$ is a directed set on which a net (taking values in $\left.m(G)^{*}\right)$ can be defined.

We define now the following net of finite means, defined on the direct set $G$ : $\left\{\phi_{h} ; h \in G\right\}$ where $\phi_{h}=Q 1_{h}$ for $h \in G$.

We will prove now that the net of finite means $\left\{\phi_{h}\right\}$ converges in norm to left invariance.

If $a \in G$ then for $f \in m(G)$ we have,

Thus

$$
\left(L_{a} \phi_{h}\right) f=\phi_{h}\left(l_{a} f\right)=\left(Q 1_{h}\right)\left(l_{a} f\right)=\left(l_{a} f\right)(h)=f(a h)=\left(Q 1_{a h}\right) f=\phi_{a h}(f) .
$$

$$
\left\|L_{a} \phi_{h}-\phi_{h}\right\|=\left\|\phi_{a h}-\phi_{h}\right\|=\left\|Q 1_{a h}-Q 1_{h}\right\|=\left\|1_{a h}-1_{h}\right\|,
$$

where the last norm is in $l_{1}(G)$. But using (5) there exists $\alpha_{0}$ such that $\alpha \geqq \alpha_{0}$ implies $a b_{\alpha}=b_{\alpha}$. Thus the element $b_{\alpha_{0}}=h_{0}$ will satisfy $a h_{0}=h_{0}$. For any $h \in G$ such that $h \geqq h_{0}$ we have either that $h=h_{0}$ and then $a h_{0}=h_{0}$, i.e., $\left\|1_{a h}-1_{h}\right\|=0$ or that $h_{0} h=h$ and then

$$
a h=a\left(h_{0} h\right)=\left(a h_{0}\right) h=h_{0} h=h,
$$

which leads again to $\left\|1_{a h}-1_{h}\right\|=0$, which shows that for any $a \in G$ there exists an element $h_{0} \in G$ (which depends on this $a$ ) such that $h \geqq h_{0}$ implies $\left\|L_{a} \phi_{h}-\phi_{h}\right\|=0$. In other words,

$$
\lim _{h}\left\|L_{a} \phi_{h}-\phi_{h}\right\|=0 \text { for any } a \in G .
$$

If $G$ as a directed set contains a finite cofinal set $\left\{a_{1}, \cdots, a_{k}\right\}$ then let $a_{0} \geqq a_{i}$ for $1 \geqq i \geqq k$. Then $\left\{a_{0}\right\}$ is also a cofinal set in the directed set $G$. In other words, $a_{0} \geqq g$ for any $g \in G$ which means that for any $g \in G$ either $a_{0}=g$ or $g a_{0}=a_{0}$ which shows that $a_{0}$ is the required element.

We will show now that the assumption that $G$ (as a directed set) does not contain a finite cofinal set contradicts our assumption that $\operatorname{dim} M l G=n<\infty$ and thus finish the proof of the theorem.

If $d \in G$ is arbitrary then there is a $d^{\prime} \in G$ such that $d^{\prime}>d$ and $d^{\prime} \neq d$, otherwise $\{d\}$ would be a finite cofinal set in $G$ (since for any $e \in G$ there is an $h \geqq e$ and $h \geqq d$ and therefore $d=h \geqq e$ ) which cannot be since we assume that $G$ has no finite cofinal subsets. We can apply now Luthar's theorem to get that for any natural number $k$ there are $k$ cofinal disjoint subsets of $G$.

Let $C_{1}, \cdots, C_{k}$ be $k$ cofinal disjoint subsets of $G$. Then $\left\{\phi_{h} ; h \in C_{i}\right\}$ as a subnet of the strongly converging to left invariance net $\left\{\phi_{h} ; h \in G\right\}$ also converges to left invariance. Let now $\phi_{i}$ be a $w^{*}$ cluster point of $\left\{\phi_{h} ; h \in C_{i}\right)$ for $i=1,2, \cdots, k$. (Since the $\phi_{h}$ are on the unit ball of $m(G)^{*}$ which is $w^{*}$ compact (see $[4$, p. 424]) there exist such $\phi_{i}$.) Moreover by Day [3, p. $\left.520(\mathrm{~B})\right], \phi_{i} \in M l(G)$.

Now $\left\{\phi_{h} ; h \in C_{i}\right\} \subset\left\{\phi \in m(G)^{*} ; \phi\left(C_{i}\right)=1\right.$ and $\phi\left(C_{j}\right)=0$ if $\left.i \neq j\right\}=A_{i}$ since for $h \in C_{i}$, 


$$
\phi_{h}\left(C_{j}\right)=\left(Q 1_{h}\right)\left(1_{C_{j}}\right)=1_{c_{j}}(h)=\left\{\begin{array}{l}
1 \text { if } j=i, \\
0 \text { if } j \neq i
\end{array}\right.
$$

(The last equality is true since the $C_{j}$ 's are disjoint.) $A_{i}$ is a $w^{*}$ closed set and therefore $\phi_{i}$, as a $w^{*}$ cluster point of $\left\{\phi_{h} ; h \in C_{i}\right\}$, belong to it. We have thus that $\phi_{1}, \cdots, \phi_{k} \in M l(G)$ satisfy

$$
\phi_{i}\left(C_{j}\right)=0 \text { if } i \neq j \text { and } \phi_{i}\left(C_{i}\right)=1 .
$$

If now $\sum_{i=1}^{k} \alpha_{i} \phi_{i}=0$ then

$$
0=\sum_{i=1}^{k} \alpha_{i} \phi_{i}\left(1_{C_{j}}\right)=\sum_{i=1}^{k} \alpha_{i} \phi_{i}\left(C_{j}\right)=\alpha_{j}
$$

Thus $\phi_{1}, \cdots, \phi_{k} \in M l(G)$ are linearly independent and $\operatorname{dim} M l(G) \geqq k$ for any natural number $k$ which cannot be since by assumption $\operatorname{dim} M l(G)=n<\infty$.

REMARKS. If $G$ has a finite group $A$ which is a (minimal) left ideal and (minimal) right ideal then $g A=A$ and thus $A$ is a (1.i.l.c.) (see $[5, \S 2]$ ). A result of Clifford (see Lemma (3.1) and Remark (3.1) in [5]) implies that the union of all the finite groups and (1.i.l.c.) in $G$ is a right minimal ideal (which contains $A$ ) and therefore coincides with $A$. Thus $A$ is the only finite group and (1.i.l.c.) (a finite group and left ideal is a minimal left ideal) and by replacing left by right we get that $A$ is also the only finite group and (r.i.r.c.). Thus by Theorem (3.1) and Remarks (3.2), (3.3) in [5]

$$
\operatorname{dim} \operatorname{Ml}(G)=\operatorname{dim} \operatorname{Mr}(G)=1 \text { and } M l(G)=\left\{\phi_{A}\right\}=\operatorname{Mr}(G)
$$

(for definition of $\phi_{A}$ see $\left.[5, \S 2]\right)$. We get thus:

COROLlaRY 3. If $G$ is a semigroup which contains a finite group which is a left ideal and right ideal then $\operatorname{dim} M l(G)=\operatorname{dim} M r(G)=1$ and $M l(G)$ $=\operatorname{Mr}(G) \subset Q l_{1}(G)$.

For commutative semigroups one has

THEOREM 3. If $G$ is a commutative semigroup then $\operatorname{dim} M l(G)$ can take the only two values 1 or $\infty$ and $\operatorname{dim} M l(G)=1$ if and only if $G$ contains a finite ideal. The second part of this theorem is a result of I. S. Luthar (see [9]).

For proof we have only to remark that commutative semigroups are amenable (see Day [3, p. 516]) and for this case $M l(G)=\operatorname{Mr}(G)$. (Compare with Corollary (5.4) in [5] and Remark (2.7) in [6].) From here we get easily the

THEOREM 4. If $G$ is a commutative semigroup with no finite ideals then the algebra $m(G) *$ has infinite dimensional radical. $(m(G) *$ stands here for the second conjugate algebra of the semigroup algebra $l_{1}(G)$.) 
In $[1$, p. 853$]$ this theorem is conjectured for commutative groups. For proof we have only to remark (as in the last footnote in [5]) that if $\phi_{0} \in M l(G)$ then $M l(G)-\phi_{0}=\left\{\phi-\phi_{0} ; \phi \in M l(G)\right\}$ is included in $J_{1}=\left\{\phi \in m(G)^{*} ; \phi(G)=0\right.$, $L_{g} \phi=\phi$ for $\left.g \in G\right\}$ which is a two-sided ideal satisfying $J_{1}^{2}=0$ (see [1,pp. 849-850]) and therefore is included in the radical.

It is interesting to note that if $G$ is an infinite commutative group then as known, $l_{1}(G)$ is a commutative semisimple algebra (i.e., with zero radical) and though $l_{1}(G)$ is $w^{*}$ dense in $m(G)^{*}$, the second conjugate algebra has an infinite dimensional radical (i.e., the radical of $l_{1}(G)$ is by no means $w^{*}$ dense in the radical of $\left.m(G)^{*}\right)$. This is even true for infinite commutative semigroups with cancellation (which are semisimple by Hewitt and Zuckerman, Trans. Amer. Math. Soc. 83 (1956), 70-97).

\section{REFERENCES}

1. P. Civin and B. Yood, The second conjugate space of a Banach algebra as an algebra, Pacific J. Math. 11 (1961), 847-870.

2. A. Clifford, Semigroups containing minimal left ideals, Amer. J. Math. 70(1948), 521-526.

3. M. M. Day, Amenable semigroups, Illinois J. Math. 1 (1957), 509-544.

4. N. Dunford and J. Schwartz, Linear operators. I, Interscience, New York, 1958.

5. E. Granirer, On amenable semigroups with a finite dimensional set of invariant means. I, Illinois J. Math. 7 (1963), 32-48.

6. - On amenable semigroups with a finite dimensional set of invariant means. II, Illinois J. Math. 7 (1963), 49-58.

7. T. Kelley, General topology, Van Nostrand, Princeton, N. J., 1955.

8. G. Köthe, Topologische Lineare Räume, Springer, Berlin, 1960.

9. I. S. Luthar, Uniqueness of the invariant mean on an abelian semigroup, Illinois J. Math. 3 (1959), 28-44.

10. E. Ljapin, Semigroups, Fizmatgiz, Moscow, 1960. (Russian)

11. R. Thibault, Séminaire Chatelet-Dubreil, Demi-groupes 1953/54, Inst. Henri Poincaré, Dissertations 13-14.

UNIVERSITY OF ILLINOIS, URBANA, ILLINOIS 